\title{
A Modified Iterative Algorithm for Split Feasibility Problems of Right Bregman Strongly Quasi-Nonexpansive Mappings in Banach Spaces with Applications
}

\author{
Anantachai Padcharoen ${ }^{1,2}$, Poom Kumam ${ }^{1,2, *}$, Yeol Je Cho ${ }^{3,4}$ and Phatiphat Thounthong ${ }^{5,6}$ \\ 1 KMUTT-Fixed Point Theory and Optimization Research Group (KMUTT-FPTO), \\ Theoretical and Computational Science Center (TaCS), Science Laboratory Building, Faculty of Science, \\ King Mongkut's University of Technology Thonburi (KMUTT), 126 Pracha-Uthit Road, Bang Mod, \\ Thrung Khru, Bangkok 10140, Thailand; apadcharoen@yahoo.com \\ 2 KMUTTFixed Point Research Laboratory, Department of Mathematics, Room SCL 802 Fixed Point \\ Laboratory, Science Laboratory Building, Faculty of Science, King Mongkut's University of Technology \\ Thonburi (KMUTT), 126 Pracha-Uthit Road, Bang Mod, Thrung Khru, Bangkok 10140, Thailand \\ 3 Department of Mathematics Education, Gyeongsang National University, Chinju 660-701, Korea; \\ yjchomath@gmail.com \\ 4 Department of Mathematics, King Abdulaziz University Jeddah 21589, Saudi Arabia \\ 5 Renewable Energy Research Centre, King Mongkut's University of Technology North Bangkok (KMUTNB), \\ Wongsawang, Bangsue, Bangkok 10800, Thailand; phtt@kmutnb.ac.th \\ 6 Department of Teacher Training in Electrical Engineering, Faculty of Technical Education, \\ King Mongkut's University of Technology North Bangkok (KMUTNB), Wongsawang, Bangsue, \\ Bangkok 10800, Thailand \\ * Correspondence: poom.kum@kmutt.ac.th
}

Academic Editor: Alicia Cordero

Received: 8 September 2016; Accepted: 1 November 2016; Published: 10 November 2016

\begin{abstract}
In this paper, we present a new iterative scheme for finding a common element of the solution set $\mathcal{F}$ of the split feasibility problem and the fixed point set $F(T)$ of a right Bregman strongly quasi-nonexpansive mapping $T$ in $p$-uniformly convex Banach spaces which are also uniformly smooth. We prove strong convergence theorem of the sequences generated by our scheme under some appropriate conditions in real $p$-uniformly convex and uniformly smooth Banach spaces. Furthermore, we give some examples and applications to illustrate our main results in this paper. Our results extend and improve the recent ones of some others in the literature.
\end{abstract}

Keywords: right Bregman strongly quasi-nonexpansive; split feasibility problem; fixed point

\section{Introduction}

Let $E_{1}, E_{2}$ be Banach spaces and $C, Q$ be nonempty closed convex subsets of $E_{1}$ and $E_{2}$, respectively. Let $A$ : $E_{1} \rightarrow E_{2}$ be a bounded linear operator. The split feasibility problem (shortly, (SFP)) is as follows:

$$
\text { Find } x \in C \text { such that } A x \in Q \text {. }
$$

We denote the solution set of the problem (SFP) by $\mathcal{F}:=\{x \in C: A x \in Q\}=C \cap A^{-1}(Q)$. It is worth mentioning that (SFP) in finite-dimensional spaces was first introduced by Censor and Elfving [1] for modelling inverse problems which arise from phase retrievals and medical image reconstruction.

Note that, in finite dimensional Hilbert spaces, the strong convergence of a sequence is equivalent to the weak convergence and the boundedness of a sequence implies that there exists a strongly 
convergent subsequence. However, in infinite dimensional Hilbert spaces, the strong convergence of a sequence is not equivalent to the weak convergence and the boundedness of a sequence implies that there exists a weakly convergent subsequence. So, for some algorithms, we can prove only strong convergence theorems in finite dimensional Hilbert spaces, but we can prove weak and strong convergence theorems in infinite dimensional Hilbert spaces.

In [2], Byrne presented a new method $\left\{x_{n}\right\}$, which is called the CQ-algorithm for solving the problem (SFP) that does not involve matrix inverses, defined as follows:

For any $x_{0} \in C$ and $n \geq 1$,

$$
x_{n+1}=P_{C}\left(x_{n}+\gamma A^{T}\left(P_{Q}-I\right) x_{n}\right),
$$

where $P_{C}$ and $P_{Q}$ is the orthogonal projections onto $C$ and $Q$, respectively, $\gamma \in\left(0, \frac{2}{L}\right), L$ is the largest eigenvalue of the matrix $A^{T} A$ and $I$ is the identity matrix.

After that many authors [3-7] study extend some iterative algorithms from Hilbert spaces to Banach spaces by using Bregman's technic as follows:

In solving the problem (SFP) in $p$-uniformly convex real Banach spaces which are also uniformly smooth, Schopfer et al. [8] proposed the following algorithm $\left\{x_{n}\right\}$ defined as follows:

For any $x_{1} \in E_{1}$ and $n \geq 1$,

$$
x_{n+1}=\Pi_{C} J_{E_{1}}^{*}\left(J_{E_{1}}\left(x_{n}\right)-s_{n} A^{*} J_{E_{2}}\left(A x_{n}-P_{Q}\left(A x_{n}\right)\right)\right),
$$

where $\Pi_{C}$ denotes the Bregman projection and $J$ the duality mapping.

Clearly, the algorithm (3) covers Byrne's CQ algorithm (2), which is a gradient-projection method (GPM) in convex minimization as a special case. The duality mapping of $E_{1}$ is sequentially weak-to-weak-continuous (see [8]) in Banach spaces such as the classical $L_{p}(2<p<\infty)$ spaces.

In [9], Wang modified the algorithm (3) and proved strong convergence theorems for the following multiple-sets split feasibility problem (MSSFP):

$$
\text { Find } x \in \bigcap_{i=1}^{r} C_{i} \text { such that } A x \in \bigcap_{j=1+r}^{r+s} Q_{j} \text {, }
$$

where $r, s$ are two given integers, $C_{i}, i=1,2,3, \cdots, r$, is a closed convex subset in $E_{1}$ and $Q_{j}, j=r+1, \cdots, r+s$, is a closed convex subset in $E_{2}$. He defined the following: for each $n \in \mathbb{N}$,

$$
T_{n}(x)= \begin{cases}\Pi_{C_{i}(n)}(x), & 1 \leq i(n) \leq r, \\ J_{E_{1}}^{*}\left[J_{E_{1}}(x)-s_{n} A^{*} J_{E_{2}}\left(A x-P_{Q_{j(n)}}(A x)\right)\right], & r+1 \leq i(n) \leq r+s,\end{cases}
$$

where $i: \mathbb{N} \rightarrow \mathcal{I}$ is the cyclic control mapping $i(n)=n \bmod (r+s)+1$ and $t_{n}$ satisfies

$$
\left.0<s \leq s_{n} \leq \frac{q}{C_{q}\|A\|^{q}}\right)^{\frac{1}{q-1}}
$$

with a constant $C_{q}$ and proposed the following algorithm $\left\{x_{n}\right\}$ defined as follows: For any $x_{1}=\bar{x}$ and $n \geq 1$,

$$
\left\{\begin{array}{l}
y_{n}=T_{n} x_{n}, \\
D_{n}=\left\{w \in E_{1}: d_{p}\left(y_{n}, w\right) \leq d_{p}\left(x_{n}, w\right)\right\}, \\
E_{n}=\left\{w \in E_{1}:\left\langle x_{n}-w, J_{p}(\bar{x})-J_{p}\left(x_{n}\right) \geq 0\right\},\right. \\
x_{n+1}=\Pi_{D_{n} \cap E_{n}}(\bar{x}) .
\end{array}\right.
$$

Recently, Zegeye and Shahzad [10] proved a strong convergence theorem for a common fixed point of a finite family of right Bregman strongly nonexpansive mappings in the framework of real 
reflexive Banach spaces. Furthermore, they applied their method to approximate a common zero of a finite family of maximal monotone operators and a solution of a finite family of convex feasibility problems in reflexive real Banach spaces.

Let $f: E \rightarrow \mathbb{R}$ be a cofinite function which is bounded, uniformly Fŕechet differentiable and totally convex on bounded subsets of $E$. Let $C$ be a nonempty closed convex subset $\operatorname{int}(\operatorname{dom} f)$ and let $T_{i}: C \rightarrow C$, for $i=1,2, \cdots, N$, be a finite family of right Bregman strongly nonexpansive mappings such that $F\left(T_{i}\right)=\widehat{F}\left(T_{i}\right)$ for each $i \in\{1,2, \cdots, N\}$. Assume that $F:=\widehat{F}\left(T_{i}\right)$ is nonempty. For any $u, x_{1} \in C$, let $\left\{x_{n}\right\}$ be a sequence generated by

$$
x_{n+1}=\alpha_{n} u+\left(1-\alpha_{n}\right) T x_{n}
$$

for each $n \geq 1$, where $T=T_{N} \circ T_{N-1} \circ \cdots \circ T_{1}$ and $\left\{\alpha_{n}\right\} \subset(0,1)$ satisfy the following conditions:

(i) $\lim _{n \rightarrow \infty} \alpha_{n}=0$;

(ii) $\sum_{n=1}^{\infty} \alpha_{n}=\infty$.

Then $\left\{x_{n}\right\}$ converges strongly to a point $\widehat{x}$.

In this paper, we modify the Halpern-Mann iterative method for split feasibility problems and fixed point problems concerning right Bregman strongly quasi-nonexpansive mappings in $p$-uniformly convex and uniformly smooth Banach spaces. We prove strong convergence theorem of the sequences generated by our scheme under some appropriate conditions in real $p$-uniformly convex and uniformly smooth Banach spaces. Also, we give numerical examples of our result to study its efficiency and implementation. Our results extend and improve the recent ones of some others in the literature.

\section{Preliminaries}

Let $E_{1}, E_{2}$ be real Banach spaces and $A: E_{1} \rightarrow E_{2}$ be a bounded linear operator. The dual (adjoint) operator of $A$, denoted by $A^{*}$, is a bounded linear operator defined by $A^{*}: E_{2}^{*} \rightarrow E_{1}^{*}$

$$
\left\langle A^{*} \bar{y}, x\right\rangle:=\langle\bar{y}, A x\rangle
$$

for all $x \in E_{1}$ and $\bar{y} \in E_{2}^{*}$ and the equalities $\left\|A^{*}\right\|=\|A\|, \mathcal{N}\left(A^{*}\right)=\mathcal{R}(A)^{\perp}$ are valid, where

$$
\mathcal{R}(A)^{\perp}:=\left\{x^{*} \in E_{2}^{*}:\left\langle x^{*}, u\right\rangle=0, \forall u \in \mathcal{R}(A)\right\} .
$$

For more details on bounded linear operators and their duals, see [11,12].

Definition 1. (1) The duality mapping $J_{E}^{p}: E \rightarrow E^{*}$ is defined by

$$
J_{E}^{p}(x)=\left\{x^{*} \in E^{*}:\left\langle x, x^{*}\right\rangle=\|x\|^{p},\left\|x^{*}\right\|=\|x\|^{p-1}\right\} .
$$

(2) The duality mapping $J_{E}^{p}$ is said to be weak-to-weak continuous if

$$
x_{n} \rightarrow x \Longrightarrow\left\langle J_{E}^{p} x_{n}, y\right\rangle \rightarrow\left\langle J_{E}^{p} x, y\right\rangle
$$

holds true for any $y \in E$.

We note here that $l_{p}(p>1)$ spaces has such a property, but the $L_{p}(p>2)$ space does not share this property. The domain of a convex function $f: E \rightarrow \mathbb{R}$ is defined by $\operatorname{dom} f:=\{x \in E: f(x)<+\infty\}$. When $\operatorname{dom} f \neq \varnothing$, then we say that $f$ is proper.

In the sequel, we adopt the following notations in this paper: $x_{n} \rightarrow x$ means that $x_{n} \rightarrow x$ strongly and $x_{n} \rightarrow x$ meansthat $x_{n} \rightarrow x$ weakly. 
Definition 2 ([13]). Let $f: E \rightarrow \mathbb{R}$ be a convex and Gâteaux differentiable function. The Bregman distance with respect to $f$ is defined by

$$
d_{f}(x, y):=f(y)-f(x)-\left\langle f^{\prime}(x), y-x\right\rangle
$$

for all $x, y \in E$.

The duality mapping $J_{E}^{p}$ is actually the derivative of the function $f_{p}(x)=\frac{1}{p}\|x\|^{p}$. If $f=f_{p}$, then the Bregman distance with respect to $f_{p}$ now becomes

$$
\begin{aligned}
d_{p}(x, y) & =\frac{1}{q}\|x\|^{p}-\left\langle J_{E}^{p} x, y\right\rangle+\frac{1}{p}\|y\|^{p} \\
& =\frac{1}{p}\left(\|y\|^{p}-\|x\|^{p}\right)+\left\langle J_{E}^{p} x, x-y\right\rangle \\
& =\frac{1}{q}\left(\|x\|^{p}-\|y\|^{p}\right)-\left\langle J_{E}^{p} x-J_{E}^{p} y, y\right\rangle .
\end{aligned}
$$

The Bregman distance is not symmetric and so it is not a metric, but it posses the following important properties: for all $w, x, y \in E$,

$$
d_{p}(x, y)=d_{p}(x, w)+d_{p}(w, y)+\left\langle w-y, J_{E}^{p} x-J_{E}^{p} y\right\rangle
$$

and

$$
d_{p}(x, y)+d_{p}(y, x)=\left\langle x-y, J_{E}^{p} x-J_{E}^{p} y\right\rangle .
$$

Let $1<q \leq 2 \leq p$ with $\frac{1}{p}+\frac{1}{q}=1$. The modulus of smoothness of $E$ is the function $\rho_{E}:[0, \infty) \rightarrow[0, \infty)$ defined by

$$
\rho_{E}(t):=\sup \left\{\frac{1}{2}(\|x+y\|+\|x-y\|)-1:\|x\| \leq 1,\|y\| \leq t\right\} .
$$

A Banach space $E$ is said to be uniformly smooth if

$$
\lim _{t \rightarrow 0} \frac{\rho_{E}(t)}{t}=0
$$

and, for any $q>1$, a Banach space $E$ is said to be $q$-uniformly smooth if there exists $C_{q}>0$ such that $\rho_{E}(t) \leq C_{q} t^{q}$ for any $t>0$.

Let $x, y \in E$ and $q>1$. If a Banach space $E$ is $q$-uniformly smooth, then there exists $C_{q}>0$ such that

$$
\|x-y\|^{q} \leq\|x\|^{q}-q\left\langle J_{E}^{p}(x), y\right\rangle+C_{q}\|y\|^{q} .
$$

Let $\operatorname{dim}(E) \geq 2$. The modulus of convexity of $E$ is the function $\delta_{E}(\epsilon):(0,2] \rightarrow[0,1]$ defined by

$$
\delta_{E}(\epsilon):=\inf \left\{1-\left\|\frac{x+y}{2}\right\|:\|x\|=\|y\|=1, \epsilon=\|x-y\|\right\} .
$$

A Banach space $E$ is said to be uniformly convex if $\delta_{E}(\epsilon)>0$ for all $\epsilon \in(0,2]$ and, for any $p>1$, a Banach space $E$ is said to be $p$-uniformly convex if there is $C_{p}>0$ such that $\delta_{E}(\epsilon) \geq C_{p} \epsilon^{p}$ for any $\epsilon \in(0,2]$. More information concerning uniformly convex spaces can be found, for example, in the book by Goebel and Reich [14].

It is known that a Banach space $E$ is $p$-uniformly convex and uniformly smooth if and only if its dual $E^{*}$ is $q$-uniformly smooth and uniformly convex. It is also well known that the duality $J_{E}^{p}$ is one-to-one, single valued and satisfies $J_{E}^{p}=\left(J_{E^{*}}^{q}\right)^{-1}$, where $J_{E^{*}}^{q}$ is the duality mapping of $E^{*}$. 
For any $p$-uniformly convex Banach space $E$, the metric and the Bregman distance have the following relation :

$$
\tau\|x-y\|^{p} \leq d_{p}(x, y) \leq\left\langle x-y, J_{E}^{p} x-J_{E}^{p} y\right\rangle,
$$

where $\tau>0$ is a fixed number.

Let $C$ be a nonempty closed convex subset of $E$. The metric projection

$$
P_{C} x:=\arg \min _{y \in C}\|x-y\|
$$

for all $x \in E$ is the unique minimizer of the norm distance, which can be characterized by a variational inequality

$$
\left\langle J_{E}^{p}\left(x-P_{C} x\right), z-P_{C} x\right\rangle \leq 0
$$

for all $z \in C$.

Similarly, the Bregman projection is defined as follows:

$$
\Pi_{C} x=\arg \min _{y \in C} d_{p}(x, y)
$$

for all $x \in E$, which is the unique minimizer of the Bregman distance. In addition, the Bregman projection can also be characterized by a variational inequality

$$
\left\langle J_{E}^{p}(x)-J_{E}^{p}\left(\Pi_{C} x\right), z-\Pi_{C} x\right\rangle \leq 0
$$

for all $z \in C$, from which one has

$$
d_{p}\left(\Pi_{C} x, z\right) \leq d_{p}(x, z)-d_{p}\left(x, \Pi_{C} x\right)
$$

for all $z \in C$.

Following [15,16], we will make use of the function $V_{p}: E^{*} \times E \rightarrow[0,+\infty)$ associated with $f_{p}$, which is defined by

$$
V_{p}(\bar{x}, x)=\frac{1}{q}\|\bar{x}\|^{q}-\langle\bar{x}, x\rangle+\frac{1}{p}\|x\|^{p}
$$

for all $x \in E$ and $\bar{x} \in E^{*}$. Then $V_{p}$ is nonnegative and

$$
V_{p}(\bar{x}, x)=d_{p}\left(J_{E}^{*}(\bar{x}), x\right)=d_{p}\left(J_{E}^{q}(\bar{x}), x\right)
$$

for all $x \in E$ and $\bar{x} \in E^{*}$. Moreover, by the subdifferential inequality, we have

$$
V_{p}(\bar{x}, x)+\left\langle\bar{y}, J_{E}^{*}(\bar{x})-x\right\rangle \leq V_{p}(\bar{x}+\bar{y}, x)
$$

for all $x \in E$ and $\bar{x}, \bar{y} \in E^{*}$ (see also $[17,18]$ ). In addition, $V_{p}$ is convex in the first variable. Thus, for all $z \in E$,

$$
d_{p}\left(J_{E}^{q}\left(\sum_{i=1}^{N} t_{i} J_{E}^{p}\left(x_{i}\right)\right), w\right)=d_{p}\left(J_{E}^{*}\left(\sum_{i=1}^{N} t_{i} J_{E}^{p}\left(x_{i}\right)\right), w\right) \leq \sum_{i=1}^{N} t_{i} d_{p}\left(x_{i}, w\right),
$$

where $\left\{x_{i}\right\} \subset E$ and $\left\{t_{i}\right\} \subset(0,1)$ with $\sum_{i=1}^{N}=1$. For more details, see $[19,20]$.

Let $C$ be a nonempty, closed and convex subset of $E$. A mapping $T: C \rightarrow C$ is said to be nonexpansive if

$$
\|T x-T y\| \leq\|x-y\|
$$

for all $x, y \in C$. We denote by $F(T)$ the set of fixed points of $T$, that is, $F(T)=\{x \in C: T x=x\}$. 
Let $C$ be a convex subset of int $\left(\operatorname{dom} f_{p}\right)$, where $f_{p}(x)=\left(\frac{1}{p}\right)\|x\|^{p}, 2 \leq p<\infty$, and $T$ be a self-mapping of $C$. A point $\widehat{x} \in C$ is called an asymptotic fixed point of $T$ if $C$ contains a sequence $\left\{x_{n}\right\}$ which converges weakly to $\widehat{x}$ and $\lim _{n \rightarrow \infty}\left\|x_{n}-T x_{n}\right\|=0$. The set of asymptotic fixed point of $T$ is denoted by $\widehat{F}(T)$ (see [21]).

In general, the Bregman distance is not a metric due to the absence of symmetry, but it has some distance-like properties.

Definition 3. A nonlinear mapping $T$ with a nonempty asymptotic fixed point set is said to be:

(1) $T$ is called right Bregman quasi-nonexpansive (shortly, $R$-BQNE) (see [22]) if $F(T) \neq \varnothing$ and

$$
d_{p}(T x, \bar{x}) \leq d_{p}(x, \bar{x})
$$

for all $x \in C$ and $\bar{x} \in F(T)$.

(2) $T$ is called right Bregman strongly quasi-nonexpansive (shortly, $R$-BSQNE) (see $[23,24]$ ) with respect to a nonempty $\widehat{F}(T)$ if

$$
d_{p}(T x, \widehat{x}) \leq d_{p}(x, \widehat{x})
$$

for all $\widehat{x} \in \widehat{F}(T), x \in C$, and if whenever $\left\{x_{n}\right\} \subset C$ is bounded, $\widehat{x} \in \widehat{F}(T)$ and $\lim _{n \rightarrow+\infty}\left(d_{p}\left(x_{n}, \widehat{x}\right)-\right.$ $\left.d_{p}\left(T x_{n}, \widehat{x}\right)\right)=0$, then it follows that $\lim _{n \rightarrow+\infty} d_{p}\left(x_{n}, T x_{n}\right)=0$.

(3) $T$ is called right Bregman firmly nonexpansive (shortly, R-BFNE) if

$$
\langle\nabla f(T x)-\nabla f(T y), T x-T y\rangle \leq\langle\nabla f(x)-\nabla f(y), T x-T y\rangle
$$

for all $x, y \in C$ or, equivalently,

$$
d_{p}(T x, T y)+d_{p}(T y, T x)+d_{p}(x, T x)+d_{p}(y, T y) \leq d_{p}(x, T y)+d_{p}(y, T x)
$$

for all $x, y \in C$.

Lemma 1 ([25]). Let $\left\{a_{n}\right\}$ be a sequence of real numbers such that there exists a nondecreasing subsequence $\left\{n_{i}\right\}$ of $\{n\}$, that is, $a_{n_{i}} \leq a_{n_{i}+1}$ for all $i \in \mathbb{N}$. Then there exists a nondecreasing subsequence $\left\{m_{k}\right\} \subset \mathbb{N}$ such that $m_{k} \rightarrow \infty$ and the following properties are satisfied for all (sufficiently large number $k \in \mathbb{N}$ ): $a_{m_{k}} \leq a_{m_{k}+1}$ and $a_{k} \leq a_{m_{k}+1}$. In fact, $m_{k}=\max \left\{j \leq k: a_{j} \leq a_{j+1}\right\}$.

Lemma 2 ([26]). Let $\left\{a_{n}\right\}$ be a sequence of nonnegative real numbers satisfying the following relation:

$$
a_{n+1} \leq\left(1-\alpha_{n}\right) a_{n}+\alpha_{n} \sigma_{n}+\gamma_{n}
$$

for each $n \geq 0$, where

(i) $\left\{\alpha_{n}\right\} \subset[0,1]$ and $\sum_{n=1}^{\infty}=\infty$;

(ii) $\limsup _{n \rightarrow \infty} \sigma_{n} \leq 0$;

(iii) $\gamma_{n} \geq 0$ and $\sum_{n=1}^{\infty} \gamma_{n}<\infty$.

Then $\lim _{n \rightarrow \infty} a_{n}=0$.

\section{Results}

Now, we give our main results in this paper. 
Theorem 1. Let $E_{1}, E_{2}$ be p-uniformly convex real Banach spaces which are also uniformly smooth and $C$, $Q$ be nonempty closed convex subsets of $E_{1}, E_{2}$, respectively. Let $A: E_{1} \rightarrow E_{2}$ be a bounded linear operator and $A^{*}: E_{2}^{*} \rightarrow E_{1}^{*}$ be the adjoint of $A$. Suppose that the problem (SFP) has a nonempty solution set $\mathcal{F}$. Let $T: C \rightarrow C$ be a right Bregman strongly quasi-nonexpansive mapping such that $F(T)=\widehat{F}(T) \neq \varnothing$ and $F(T) \cap \mathcal{F} \neq \varnothing$. Suppose that $\left\{\alpha_{n}\right\},\left\{\beta_{n}\right\},\left\{\gamma_{n}\right\}$ and $\left\{\delta_{n}\right\}$ are the sequences in $(0,1)$ such that $\alpha_{n}+\beta_{n}+\gamma_{n}+\delta_{n}=1$, $\alpha_{n} \leq b<1,\left(1-\alpha_{n}\right) a<\gamma_{n}<\delta_{n}, a \in\left(0, \frac{1}{2}\right)$. For any fixed $u \in C$, let the sequences $\left\{x_{n}\right\}$ and $\left\{u_{n}\right\}$ be iteratively generated by $u_{0} \in E_{1}$ and

$$
\left\{\begin{array}{l}
x_{n}=\Pi_{C} J_{E_{1}^{*}}^{q}\left(J_{E_{1}}^{p}\left(u_{n}\right)-s_{n} A^{*} J_{E_{2}}^{p}\left(I-P_{Q}\right) A u_{n}\right), \\
u_{n+1}=\Pi_{C} J_{E_{1}^{*}}^{q}\left(\alpha_{n} J_{E_{1}}^{p}(u)+\left(1-\alpha_{n}\right)\left(\beta_{n} J_{E_{1}}^{p}(u)+\gamma_{n} J_{E_{1}}^{p}\left(x_{n}\right)+\delta_{n} J_{E_{1}}^{p}\left(T x_{n}\right)\right)\right)
\end{array}\right.
$$

for each $n \geq 1$. Suppose the following conditions are satisfied:
(a) $\lim _{n \rightarrow \infty} \alpha_{n}=0$;
(b) $\sum_{n=1}^{\infty} \alpha_{n}=\infty$;
(c) $0<s \leq s_{n} \leq k<\left(\frac{q}{C_{q}\|A\|^{q}}\right)^{\frac{1}{q-1}}$.

Then the sequence $\left\{x_{n}\right\}$ and $\left\{u_{n}\right\}$ converge strongly to a point $\Pi_{F(T) \cap \mathcal{F}} \mathcal{u}$.

Proof. Firstly, we prove that the sequences $\left\{x_{n}\right\}$ and $\left\{u_{n}\right\}$ are bounded. Setting $z_{n}:=A u_{n}-P_{Q}\left(A u_{n}\right)$ for each $n \geq 1$. From (14), it follows that, for any $w \in F(T) \cap \mathcal{F}$,

$$
\begin{aligned}
\left\langle J_{E_{2}}^{p}\left(z_{n}\right), A u_{n}-A w\right\rangle & =\left\|A u_{n}-P_{Q}\left(A u_{n}\right)\right\|^{p}+\left\langle J_{E_{2}}^{p}\left(z_{n}\right), P_{Q}\left(A u_{n}\right)-A w\right\rangle \\
& \geq\left\|A u_{n}-P_{Q}\left(A u_{n}\right)\right\|^{p} \\
& =\left\|z_{n}\right\|^{p} .
\end{aligned}
$$

So, from (21) and (12), it follows that

$$
\begin{aligned}
& d_{p}\left(x_{n}, w\right) \\
& \leq d_{p}\left(J_{E_{1}^{*}}^{q}\left[J_{E_{1}}^{p}\left(u_{n}\right)-s_{n} A^{*} J_{E_{2}}^{p}\left(I-P_{Q}\right) A u_{n}\right], w\right) \\
& =\frac{1}{q}\left\|J_{E_{1}}^{p}\left(u_{n}\right)-s_{n} A^{*} J_{E_{2}}^{p}\left(z_{n}\right)\right\|^{q}-\left\langle J_{E_{1}}^{p}\left(u_{n}\right), w\right\rangle+s_{n}\left\langle J_{E_{2}}^{p}\left(z_{n}\right), A w\right\rangle+\frac{1}{p}\|w\|^{p} \\
& \leq\left\|J_{E_{1}}^{p}\left(u_{n}\right)\right\|-s_{n}\left\langle A u_{n}, J_{E_{2}}^{p}\left(z_{n}\right)\right\rangle+\frac{C_{q}\left(s_{n}\|A\|\right)^{q}}{q}\left\|J_{E_{2}}^{p}\left(z_{n}\right)\right\|^{p} \\
& -\left\langle J_{E_{1}}^{p}\left(u_{n}\right), w\right\rangle+\frac{1}{p}\|w\|^{p}+s_{n}\left\langle A w, J_{E_{2}}^{p}\left(z_{n}\right)\right\rangle \\
& =\frac{1}{q}\left\|u_{n}\right\|^{p}-\left\langle J_{E_{1}}^{p}\left(u_{n}\right), w\right\rangle+\frac{1}{q}\|w\|^{p}+s_{n}\left\langle J_{E_{2}}^{p}\left(z_{n}\right), A w-A u_{n}\right\rangle \\
& +\frac{C_{q}\left(s_{n}\|A\|\right)^{q}}{q}\left\|z_{n}\right\|^{p} \\
& =d_{p}\left(u_{n}, w\right)+s_{n}\left\langle J_{E_{2}}^{p}\left(z_{n}\right), A w-A u_{n}\right\rangle+\frac{C_{q}\left(s_{n}\|A\|\right)^{q}}{q}\left\|z_{n}\right\|^{p} \\
& \leq d_{p}\left(u_{n}, w\right)-\left(s_{n}-\frac{C_{q}\left(s_{n}\|A\|\right)^{q}}{q}\right)\left\|z_{n}\right\|^{p} .
\end{aligned}
$$

By using $(c)$, we obtain

$$
d_{p}\left(x_{n}, w\right) \leq d_{p}\left(u_{n}, w\right)
$$


From (20), we have

$$
\begin{aligned}
d_{p} & \left(u_{n+1}, w\right) \\
\leq & d_{p}\left(J_{E_{1}^{*}}^{q}\left[\alpha_{n} J_{E_{1}}^{p}(u)+\left(1-\alpha_{n}\right)\left(\beta_{n} J_{E_{1}}^{p}(u)+\gamma_{n} J_{E_{1}}^{p}\left(x_{n}\right)+\delta_{n} J_{E_{1}}^{p}\left(T x_{n}\right)\right)\right], w\right) \\
= & d_{p}\left(J _ { E _ { 1 } ^ { * } } ^ { q } \left[\alpha_{n} J_{E_{1}}^{p}(u)+\left(1-\alpha_{n}\right) \beta_{n} J_{E_{1}}^{p}(u)\right.\right. \\
& \left.\left.+\left(1-\alpha_{n}\right) \gamma_{n} J_{E_{1}}^{p}\left(x_{n}\right)+\left(1-\alpha_{n}\right) \delta_{n} J_{E_{1}}^{p}\left(T x_{n}\right)\right], w\right) \\
\leq & \alpha_{n} d_{p}(u, w)+\left(1-\alpha_{n}\right) \beta_{n} d_{p}(u, w) \\
& +\left(1-\alpha_{n}\right) \gamma_{n} d_{p}\left(x_{n}, w\right)+\left(1-\alpha_{n}\right) \delta_{n} d_{p}\left(T x_{n}, w\right) \\
\leq & \alpha_{n} d_{p}(u, w)+\left(1-\alpha_{n}\right) \beta_{n} d_{p}(u, w) \\
& +\left(1-\alpha_{n}\right) \gamma_{n} d_{p}\left(x_{n}, w\right)+\left(1-\alpha_{n}\right) \delta_{n} d_{p}\left(x_{n}, w\right) \\
= & \left(\alpha_{n}+\left(1-\alpha_{n}\right) \beta_{n}\right) d_{p}(u, w)+\left(1-\alpha_{n}\right)\left(\gamma_{n}+\delta_{n}\right) d_{p}\left(x_{n}, w\right) \\
\leq & \left(\alpha_{n}+\left(1-\alpha_{n}\right) \beta_{n}\right) d_{p}(u, w)+\left(1-\alpha_{n}\right)\left(\gamma_{n}+\delta_{n}\right) d_{p}\left(u_{n}, w\right) \\
\leq & \max \left\{d_{p}(u, w), d_{p}\left(u_{n}, w\right)\right\} \\
& \ldots \\
\leq & \max \left\{d_{p}(u, w), d_{p}\left(u_{1}, w\right)\right\} .
\end{aligned}
$$

Thus $\left\{d_{p}\left(u_{n}, w\right)\right\}$ is bounded and, consequently, we have that $\left\{d_{p}\left(x_{n}, w\right)\right\}$ is bounded. Hence the sequence $\left\{x_{n}\right\}$ and $\left\{u_{n}\right\}$ are bounded. Setting

$$
y_{n}=J_{E_{1}^{*}}^{q}\left[\alpha_{n} J_{E_{1}}^{p}(u)+(1-\alpha)\left(\beta_{n} J_{E_{1}}^{p}(u)+\gamma_{n} J_{E_{1}}^{p}\left(x_{n}\right)+\delta_{n} J_{E_{1}}^{p}\left(T x_{n}\right)\right)\right]
$$

for each $n \geq 1$. Then we have

$$
\begin{aligned}
& d_{p}\left(x_{n+1}, w\right) \\
& \leq d_{p}\left(u_{n+1}, w\right) \\
& \leq d_{p}\left(J_{E_{1}^{*}}^{q}\left[\alpha_{n} J_{E_{1}}^{p}(u)+\left(1-\alpha_{n}\right)\left(\beta_{n} J_{E_{1}}^{p}(u)+\gamma_{n} J_{E_{1}}^{p}\left(x_{n}\right)+\delta_{n} J_{E_{1}}^{p}\left(T x_{n}\right)\right)\right], w\right) \\
&=V_{f}\left(\alpha_{n} J_{E_{1}}^{p}(u)+\left(1-\alpha_{n}\right)\left(\beta_{n} J_{E_{1}}^{p}(u)+\gamma_{n} J_{E_{1}}^{p}\left(x_{n}\right)+\delta_{n} J_{E_{1}}^{p}\left(T x_{n}\right)\right), w\right) \\
& \leq V_{f}\left(\alpha_{n} J_{E_{1}}^{p}(u)+\left(1-\alpha_{n}\right)\left(\beta_{n} J_{E_{1}}^{p}(u)+\gamma_{n} J_{E_{1}}^{p}\left(x_{n}\right)+\delta_{n} J_{E_{1}}^{p}\left(T x_{n}\right)\right)\right. \\
&\left.-\alpha_{n}\left(J_{E_{1}}^{p}(u)-J_{E_{1}}^{p}(w)\right), w\right)-\left\langle-\alpha_{n}\left(J_{E_{1}}^{p}(u)-J_{E_{1}}^{p}(w)\right),\right. \\
&\left.J_{E_{1}^{*}}^{q}\left[\alpha_{n} J_{E_{1}}^{p}(u)+\left(1-\alpha_{n}\right)\left(\beta_{n} J_{E_{1}}^{p}(u)+\gamma_{n} J_{E_{1}}^{p}\left(x_{n}\right)+\delta_{n} J_{E_{1}}^{p}\left(T x_{n}\right)\right)\right]-w\right\rangle \\
&= V_{f}\left(\alpha_{n} J_{E_{1}}^{p}(u)+\left(1-\alpha_{n}\right)\left(\beta_{n} J_{E_{1}}^{p}(u)+\gamma_{n} J_{E_{1}}^{p}\left(x_{n}\right)+\delta_{n} J_{E_{1}}^{p}\left(T x_{n}\right)\right), w\right) \\
&+\alpha_{n}\left\langle J_{E_{1}}^{p}(u)-J_{E_{1}}^{p}(w), y_{n}-w\right\rangle \\
&= V_{f}\left(\alpha_{n} J_{E_{1}}^{p}(w)+\left(1-\alpha_{n}\right)\left(\beta_{n} J_{E_{1}}^{p}(u)+\gamma_{n} J_{E_{1}}^{p}\left(x_{n}\right)+\delta_{n} J_{E_{1}}^{p}\left(T x_{n}\right)\right), w\right) \\
&+\alpha_{n}\left\langle J_{E_{1}}^{p}(u)-J_{E_{1}}^{p}(w), y_{n}-w\right\rangle \\
&= d_{p}\left(J_{E_{1}^{*}}^{q}\left[\alpha_{n} J_{E_{1}}^{p}(w)+\left(1-\alpha_{n}\right)\left(\beta_{n} J_{E_{1}}^{p}(u)+\gamma_{n} J_{E_{1}}^{p}\left(x_{n}\right)+\delta_{n} J_{E_{1}}^{p}\left(T x_{n}\right)\right)\right], w\right) \\
&+\alpha_{n}\left\langle n J_{E_{1}}^{p}(u)-J_{E_{1}}^{p}(w), y_{n}-w\right\rangle \\
& \leq \alpha_{n} d_{p}(w, w)+\left(1-\alpha_{n}\right) \beta_{n} d_{p}(w, w)+\left(1-\alpha_{n}\right) \gamma_{n} d_{p}\left(x_{n}, w\right) \\
&+\left(1-\alpha_{n}\right) \delta_{n} d_{p}\left(T x_{n}, w\right)
\end{aligned}
$$




$$
\begin{aligned}
\leq & \left(1-\alpha_{n}\right) \gamma_{n} d_{p}\left(x_{n}, w\right)+\left(1-\alpha_{n}\right) \delta_{n} d_{p}\left(x_{n}, w\right) \\
& +\alpha_{n}\left\langle J_{E_{1}}^{p}(u)-J_{E_{1}}^{p}(w), y_{n}-w\right\rangle \\
= & \left(1-\alpha_{n}\right)\left(\gamma_{n}+\delta_{n}\right) d_{p}\left(u_{n}, w\right)+\alpha_{n}\left\langle J_{E_{1}}^{p}(u)-J_{E_{1}}^{p}(w), y_{n}-w\right\rangle \\
\leq & \left(1-\alpha_{n}\right) d_{p}\left(u_{n}, w\right)+\alpha_{n}\left\langle J_{E_{1}}^{p}(u)-J_{E_{1}}^{p}(w), y_{n}-w\right\rangle .
\end{aligned}
$$

Now, we prove the strong convergence theorem by the two cases:

Case I. Suppose that there exists $n_{0} \in \mathbb{N}$ such that $\left\{d_{p}\left(x_{n}, w\right)\right\}$ is monotonically non-increasing for all $n \geq n_{0}$. Then $\left\{d_{p}\left(x_{n}, w\right)\right\}$ converges and, as $n \rightarrow \infty$,

$$
d_{p}\left(x_{n+1}, w\right)-d_{p}\left(x_{n}, w\right) \rightarrow 0 .
$$

Setting $t_{n}=J_{E_{1}^{*}}^{q}\left(\frac{\beta_{n}}{1-\alpha_{n}} J_{E_{1}}^{p}\left(x_{n}\right)+\frac{\gamma_{n}}{1-\alpha_{n}} J_{E_{1}}^{p}\left(x_{n}\right)+\frac{\delta_{n}}{1-\alpha_{n}} J_{E_{1}}^{p}\left(T x_{n}\right)\right)$. Then we have

$$
\begin{aligned}
d_{p}\left(t_{n}, w\right) & =d_{p}\left(J_{E_{1}^{*}}^{q}\left(\frac{\gamma_{n}}{1-\alpha_{n}} J_{E_{1}}^{p}\left(x_{n}\right)+\frac{\delta_{n}}{1-\alpha_{n}} J_{E_{1}}^{p}\left(T x_{n}\right)\right), w\right) \\
& \leq \frac{\gamma_{n}}{1-\alpha_{n}} d_{p}\left(x_{n}, w\right)+\frac{\delta_{n}}{1-\alpha_{n}} d_{p}\left(T x_{n}, w\right) \\
& \leq \frac{\gamma_{n}+\delta_{n}}{1-\alpha_{n}} d_{p}\left(x_{n}, w\right) \\
& \leq d_{p}\left(x_{n}, w\right) .
\end{aligned}
$$

Therefore, we have

$$
\begin{aligned}
0 & \leq d_{p}\left(x_{n}, w\right)-d_{p}\left(t_{n}, w\right) \\
& =d_{p}\left(x_{n}, w\right)-d_{p}\left(x_{n+1}, w\right)+d_{p}\left(x_{n+1}, w\right)-d_{p}\left(t_{n}, w\right) \\
& \leq d_{p}\left(x_{n}, w\right)-d_{p}\left(x_{n+1}, w\right)+d_{p}\left(u_{n+1}, w\right)-d_{p}\left(t_{n}, w\right) \\
& \leq d_{p}\left(x_{n}, w\right)-d_{p}\left(x_{n+1}, w\right)+\alpha_{n} d_{p}(u, w)+\left(1-\alpha_{n}\right) d_{p}\left(t_{n}, w\right)-d_{p}\left(t_{n}, w\right) \\
& =d_{p}\left(x_{n}, w\right)-d_{p}\left(x_{n+1}, w\right)+\alpha_{n} d_{p}(u, w)-\alpha_{n} d_{p}\left(t_{n}, w\right) \rightarrow 0
\end{aligned}
$$

as $n \rightarrow \infty$. Again, we obtain

$$
\begin{aligned}
d_{p}\left(t_{n}, w\right) & \leq \frac{\gamma_{n}}{1-\alpha_{n}} d_{p}\left(x_{n}, w\right)+\frac{\delta_{n}}{1-\alpha_{n}} d_{p}\left(T x_{n}, w\right) \\
& =\left(1-\frac{\beta_{n}+\delta_{n}}{1-\alpha_{n}}\right) d_{p}\left(x_{n}, w\right)+\frac{\delta_{n}}{1-\alpha_{n}} d_{p}\left(T x_{n}, w\right) \\
& =d_{p}\left(x_{n}, w\right)-\frac{\beta_{n}}{1-\alpha_{n}} d_{p}\left(x_{n}, w\right)+\frac{\delta_{n}}{1-\alpha_{n}}\left(d_{p}\left(T x_{n}, w\right)-d_{p}\left(x_{n}, w\right)\right) \\
& \leq d_{p}\left(x_{n}, w\right)+\frac{\delta_{n}}{1-\alpha_{n}}\left(d_{p}\left(T x_{n}, w\right)-d_{p}\left(x_{n}, w\right)\right) .
\end{aligned}
$$

Since $\alpha_{n}+\delta_{n} \leq 1$ and $\alpha_{n} \leq b<1$, we have

$$
\begin{aligned}
a\left(d_{p}\left(x_{n}, w\right)-d_{p}\left(T x_{n}, w\right)\right) & <\frac{\delta_{n}}{1-\alpha_{n}}\left(d_{p}\left(x_{n}, w\right)-d_{p}\left(T x_{n}, w\right)\right) \\
& \leq d_{p}\left(x_{n}, w\right)-d_{p}\left(t_{n}, w\right) \rightarrow 0
\end{aligned}
$$

as $n \rightarrow \infty$. By using $(c)$, we have

$$
d_{p}\left(x_{n}, w\right)-d_{p}\left(T x_{n}, w\right) \rightarrow 0
$$


as $n \rightarrow \infty$. Since $T$ is right Bregman strongly quasi-nonexpansive, we obtain

$$
\lim _{n \rightarrow \infty} d_{p}\left(T x_{n}, x_{n}\right)=0,
$$

which implies that

$$
\lim _{n \rightarrow \infty}\left\|T x_{n}-x_{n}\right\|=0
$$

Since $\left\{x_{n}\right\}$ is bounded and $E$ is reflexive, there exists a subsequence $\left\{x_{n_{i}}\right\}$ of $\left\{x_{n}\right\}$ which converges weakly to $\bar{x} \in C$. From (30), it follows that $\bar{x} \in F(T)$ since $F(T)=\widehat{F}(T)$.

Next, we prove that $A \bar{x} \in Q$, that is, $\bar{x} \in \mathcal{F}$. Setting

$$
v_{n}=J_{E_{1}^{*}}^{q}\left[J_{E_{1}}^{p}\left(u_{n}\right)-s_{n} A^{*} J_{E_{2}}^{p}\left(I-P_{Q}\right) A u_{n}\right] .
$$

From (16), (22) and (24), it follows that

$$
\begin{aligned}
d_{p}\left(v_{n}, x_{n}\right) & =d_{p}\left(v_{n}, \Pi_{c} v_{n}\right) \\
& \leq d_{p}\left(v_{n}, w\right)-d_{p}\left(x_{n}, w\right) \\
& \left.\leq d_{(} u_{n}, w\right)-d_{p}\left(x_{n}, w\right) \\
& \leq \alpha_{n} \mathcal{M}+d_{p}\left(x_{n-1}, w\right)-d_{p}\left(x_{n}, w\right) \rightarrow 0
\end{aligned}
$$

as $n \rightarrow \infty$, where $\mathcal{M}>0$ and $d_{p}\left(x_{n-1}, w\right)+\left\langle J_{E_{1}}^{p}(u)-J_{E_{1}}^{p}(w), y_{n-1}-w\right\rangle \leq \mathcal{M}$. Hence we have

$$
\lim _{n \rightarrow \infty}\left\|x_{n}-v_{n}\right\|=0 .
$$

From (22), it follows that, as $n \rightarrow \infty$,

$$
\begin{aligned}
\left(s_{n}-\frac{C_{q}\left(s_{n}\|A\|\right)^{q}}{q}\right)\left\|z_{n}\right\|^{p} & \leq d\left(u_{n}, w\right)-d_{p}\left(x_{n}, w\right) \\
& \leq \alpha_{n} \mathcal{M}+d_{p}\left(x_{n-1}, w\right)-d_{p}\left(x_{n}, w\right) \rightarrow 0 .
\end{aligned}
$$

Since

$$
s\left(1-\frac{C_{q} k^{q-1}(\|A\|)^{q}}{q}\right) \leq\left(s_{n}-\frac{C_{q}\left(s_{n}\|A\|\right)^{q}}{q}\right),
$$

it follows that $\left\|z_{n}\right\|^{p} \rightarrow 0$ as $n \rightarrow \infty$, which implies that $\left\|A u_{n}-P_{Q}\left(u_{n}\right)\right\| \rightarrow 0$ as $n \rightarrow \infty$. By the definition of $v_{n}$, we have

$$
\begin{aligned}
0 & \leq \| J_{E_{1}}^{p}\left(v_{n}\right)-J_{E_{1}}^{p}\left(u_{n}\right) \\
& \leq s_{n}\left\|A^{*}\right\|\left\|J_{E_{2}}^{p}\left(A u_{n}-P_{Q}\left(A u_{n}\right)\right)\right\| \\
& \leq\left(\frac{q}{C_{q}\|A\|^{q}}\right)^{\frac{1}{q-1}}\left\|A^{*}\right\|\left\|A u_{n}-P_{Q}\left(A u_{n}\right)\right\| \rightarrow 0
\end{aligned}
$$

as $n \rightarrow \infty$. Since $J_{E_{1}^{*}}^{q}$ is norm to norm uniformly continuous on bounded subsets of $E_{1}^{*}$, we obtain

$$
\lim _{n \rightarrow \infty}\left\|v_{n}-u_{n}\right\|=\lim _{n \rightarrow \infty}\left\|J_{E_{1}^{*}}^{q}\left(J_{E_{1}}^{p}\left(v_{n}\right)\right)-J_{E_{1}^{*}}^{q}\left(J_{E_{1}}^{p}\left(u_{n}\right)\right)\right\| \rightarrow 0
$$

as $n \rightarrow \infty$. From (3) and (36), we obtain

$$
\left\|x_{n}-u_{n}\right\| \leq\left\|x_{n}-y_{n}\right\|+\left\|y_{n}-u_{n}\right\| \rightarrow 0 .
$$


as $n \rightarrow \infty$. From (14), it follows that

$$
\begin{aligned}
& \left\|\left(I-P_{Q}\right) A \bar{x}\right\|^{p} \\
& =\left\langle J_{E_{2}}^{p}\left(A \bar{x}-P_{Q} A \bar{x}\right), A \bar{x}-P_{Q} A \bar{x}\right\rangle \\
& =\left\langle J_{E_{2}}^{p}\left(A \bar{x}-P_{Q} A \bar{x}\right), A \bar{x}-A u_{n_{i}}\right\rangle+\left\langle J_{E_{2}}^{p}\left(A \bar{x}-P_{Q} A \bar{x}\right), A u_{n_{i}}-P_{Q} A u_{n_{i}}\right\rangle \\
& \quad+\left\langle J_{E_{2}}^{p}\left(A \bar{x}-P_{Q} A \bar{x}\right), P_{Q} A u_{n_{i}}-P_{Q} A \bar{x}\right\rangle \\
& \leq\left\langle J_{E_{2}}^{p}\left(A \bar{x}-P_{Q} A \bar{x}\right), A \bar{x}-A u_{n_{i}}\right\rangle+\left\langle J_{E_{2}}^{p}\left(A \bar{x}-P_{Q} A \bar{x}\right), A u_{n_{i}}-P_{Q} A u_{n_{i}}\right\rangle .
\end{aligned}
$$

By the continuity of $A$ and $\left\|x_{n}-u_{n}\right\| \rightarrow 0$ as $n \rightarrow \infty$, we have $A u_{n_{i}} \rightarrow A \bar{x}$ as $n \rightarrow \infty$. Thus, letting $i \rightarrow \infty$, we have

$$
\left\|A \bar{x}-P_{Q} A \bar{x}\right\|=0 .
$$

Hence $A \bar{x}=P_{Q} A \bar{x}$, that is, $A \bar{x} \in Q$. Therefore, we have that $\bar{x} \in F(T) \cap \mathcal{F}$.

Next, we prove that $\left\{x_{n}\right\}$ converges strongly to $\Pi_{P(T) \cap \mathcal{F}} \mathcal{u}$. Now, we have

$$
\begin{aligned}
& d_{p}\left(y_{n}, x_{n}\right) \\
& =d_{p}\left(J_{E_{1}^{*}}^{q}\left[\alpha_{n} J_{E_{1}}^{p}(u)+\left(1-\alpha_{n}\right)\left(\beta_{n} J_{E_{1}}^{p}(u)+\gamma_{n} J_{E_{1}}^{p}\left(x_{n}\right)+\delta_{n} J_{E_{1}}^{p}\left(T x_{n}\right)\right)\right], x_{n}\right) \\
& \leq \alpha_{n} d_{p}\left(u, x_{n}\right)+\left(1-\alpha_{n}\right) \beta_{n} d_{p}\left(u, x_{n}\right) \\
& \quad+\left(1-\alpha_{n}\right) \gamma_{n} d_{p}\left(x_{n}, x_{n}\right)+(1-\alpha) \delta_{n} d_{p}\left(T x_{n}, x_{n}\right) \\
& \rightarrow 0
\end{aligned}
$$

as $n \rightarrow \infty$. Thus we have

$$
\left\|y_{n}-x_{n}\right\| \rightarrow 0
$$

as $n \rightarrow \infty$. Let $\widehat{x}=\Pi_{P(T) \cap \mathcal{F}} u$. From (24), we have

$$
d_{p}\left(x_{n+1}, \widehat{x}\right) \leq\left(1-\alpha_{n}\right) d_{p}\left(x_{n}, \widehat{x}\right)+\alpha_{n}\left\langle J_{E_{1}}^{p}(u)-J_{E_{1}}^{p}(\widehat{x}), y_{n}-\widehat{x}\right\rangle .
$$

Then there exists a subsequence $\left\{x_{n_{j}}\right\}$ of $\left\{x_{n}\right\}$ such that

$$
\limsup _{n \rightarrow \infty}\left\langle J_{E_{1}}^{p}(u)-J_{E_{1}}^{p}(\widehat{x}), x_{n}-\widehat{x}\right\rangle=\lim _{j \rightarrow \infty}\left\langle J_{E_{1}}^{p}(u)-J_{E_{1}}^{p}(\widehat{x}), x_{n_{j}}-\widehat{x}\right\rangle
$$

and $x_{n_{j}} \rightarrow \bar{x}$. Thus, from (15), it follows that

$$
\begin{aligned}
\limsup _{n \rightarrow \infty}\left\langle J_{E_{1}}^{p}(u)-J_{E_{1}}^{p}(\widehat{x}), x_{n}-\widehat{x}\right\rangle & =\lim _{j \rightarrow \infty}\left\langle J_{E_{1}}^{p}(u)-J_{E_{1}}^{p}(\widehat{x}), x_{n_{j}}-\widehat{x}\right\rangle \\
& =\left\langle J_{E_{1}}^{p}(u)-J_{E_{1}}^{p}(\widehat{x}), \bar{x}-\widehat{x}\right\rangle \\
& \leq 0 .
\end{aligned}
$$

Since $\left\|x_{n}-y_{n}\right\| \rightarrow 0$ as $n \rightarrow \infty$, we have

$$
\limsup _{n \rightarrow \infty}\left\langle J_{E_{1}}^{p}(u)-J_{E_{1}}^{p}(\widehat{x}), y_{n}-\widehat{x}\right\rangle \leq 0
$$

Hence, by Lemma 2, we conclude that $d_{p}\left(x_{n}, \widehat{x}\right) \rightarrow 0$ as $n \rightarrow \infty$. Therefore, $x_{n} \rightarrow \widehat{x}$ as $n \rightarrow \infty$ and, since $\left\|x_{n}-u_{n}\right\| \rightarrow 0$ as $n \rightarrow \infty$, we have $u_{n} \rightarrow \widehat{x}$ as $n \rightarrow \infty$.

Case II. Suppose that there exists a subsequence $\left\{n_{j}\right\}$ of $\{n\}$ such that

$$
d_{p}\left(x_{n_{i}}, \widehat{x}\right)<d_{p}\left(x_{n_{i}+1}, \widehat{x}\right)
$$


for all $j \in \mathbb{N}$. Then, by Lemma 1 , there exists a nondecreasing sequence $\left\{m_{k}\right\} \subset \mathbb{N}$ with $m_{k} \rightarrow \infty$ as $n \rightarrow \infty$ such that

$$
d_{p}\left(x_{m_{k}}, \widehat{x}\right) \leq d_{p}\left(x_{m_{k}+1}, \widehat{x}\right), \quad d_{p}\left(x_{k}, \widehat{x}\right) \leq d_{p}\left(x_{m_{k}+1}, \widehat{x}\right)
$$

for all $k \in \mathbb{N}$. Thus it follows from (27) and the same methods in the proof of Case I that

$$
\left\|x_{m_{k}+1}-x_{m_{k}}\right\| \rightarrow 0, \quad\left\|T x_{m_{k}}-x_{m_{k}}\right\| \rightarrow 0
$$

as $k \rightarrow \infty$. Therefore, we have

$$
\limsup _{k \rightarrow \infty}\left\langle J_{E_{1}}^{p}(u)-J_{E_{1}}^{p}(\widehat{x}), y_{m_{k}+1}-\widehat{x}\right\rangle=\limsup _{k \rightarrow \infty}\left\langle J_{E_{1}}^{p}(u)-J_{E_{1}}^{p}(\widehat{x}), y_{m_{k}}-\widehat{x}\right\rangle \leq 0 .
$$

From (24), also, we have

$$
d_{p}\left(x_{m_{k}+1}, \widehat{x}\right) \leq\left(1-\alpha_{m_{k}}\right) d_{p}\left(x_{m_{k}}, \widehat{x}\right)+\alpha_{m_{k}}\left\langle J_{E_{1}}^{p}(u)-J_{E_{1}}^{p}(\widehat{x}), y_{m_{k}}-\widehat{x}\right\rangle .
$$

Since $d_{p}\left(x_{m_{k}}, \widehat{x}\right) \leq d_{p}\left(x_{m_{k}+1}, \widehat{x}\right)$, it follows from (40) that

$$
\begin{aligned}
\alpha_{m_{k}} d_{p}\left(x_{m_{k}}, \widehat{x}\right) & \leq d_{p}\left(x_{m_{k}}, \widehat{x}\right)-d_{p}\left(x_{m_{k}+1}, \widehat{x}\right)+\alpha_{m_{k}}\left\langle J_{E_{1}}^{p}(u)-J_{E_{1}}^{p}(\widehat{x}), y_{m_{k}}-\widehat{x}\right\rangle \\
& \leq \alpha_{m_{k}}\left\langle J_{E_{1}}^{p}(u)-J_{E_{1}}^{p}(\widehat{x}), y_{m_{k}}-\widehat{x}\right\rangle
\end{aligned}
$$

Since $\alpha_{m_{k}}>0$, we obtain

$$
d_{p}\left(x_{m_{k}}, \widehat{x}\right) \leq\left\langle J_{E_{1}}^{p}(u)-J_{E_{1}}^{p}(\widehat{x}), y_{m_{k}}-\widehat{x}\right\rangle .
$$

Then, from (39), it follows that $d_{p}\left(x_{m_{k}}, \widehat{x}\right) \rightarrow 0$ as $k \rightarrow \infty$. This together with (40), we obtain $d_{p}\left(x_{m_{k}+1}, \widehat{x}\right) \rightarrow 0$ as $k \rightarrow \infty$. Since $d_{p}\left(x_{k}, \widehat{x}\right) \leq\left(x_{m_{k}+1}, \widehat{x}\right)$ for all $k \in \mathbb{N}$, we have $x_{k} \rightarrow \widehat{x}$ as $k \rightarrow \infty$, which implies that $x_{n} \rightarrow \widehat{x}$ as $k \rightarrow \infty$.

Therefore, from the above two cases, we conclude that $\left\{x_{n}\right\}$ converges strongly to $\widehat{x}=\Pi_{P(T) \cap \mathcal{F}} u$. This completes the proof.

Corollary 1 ([19]). Let $E_{1}, E_{2}$ be two p-uniformly convex real Banach spaces which are also uniformly smooth and $C, Q$ be nonempty closed convex subsets of $E_{1}, E_{2}$, respectively. Let $A: E_{1} \rightarrow E_{2}$ be a bounded linear operator and $A^{*}: E_{2}^{*} \rightarrow E_{1}^{*}$ be the adjoint of $A$. Suppose that the problem (SFP) has a nonempty solution set $\mathcal{F}$. Let $T: C \rightarrow C$ be a right Bregman strongly quasi-nonexpansive mapping such that $F(T)=\widehat{F}(T) \neq \varnothing$ and $F(T) \cap \mathcal{F} \neq \varnothing$. Suppose that $\left\{\beta_{n}\right\},\left\{\gamma_{n}\right\}$ and $\left\{\delta_{n}\right\}$ are the sequences in $(0,1)$ such that $\beta_{n}+\gamma_{n}+\delta_{n}=1$, $\beta_{n} \leq b<1,\left(1-\beta_{n}\right) a<\gamma_{n}<\delta_{n}, a \in\left(0, \frac{1}{2}\right)$. For any fixed $u \in C$, let the sequences $\left\{x_{n}\right\}$ and $\left\{u_{n}\right\}$ be iteratively generated by $u_{0} \in E_{1}$ and

$$
\left\{\begin{array}{l}
x_{n}=\Pi_{C} J_{E_{1}^{*}}^{q}\left(J_{E_{1}}^{p}\left(u_{n}\right)-s_{n} A^{*} J_{E_{2}}^{p}\left(I-P_{Q}\right) A u_{n}\right), \\
u_{n+1}=\Pi_{C} J_{E_{1}^{*}}^{q}\left(\beta_{n} J_{E_{1}}^{p}(u)+\gamma_{n} J_{E_{1}}^{p}\left(x_{n}\right)+\delta_{n} J_{E_{1}}^{p}\left(T x_{n}\right)\right)
\end{array}\right.
$$

for each $n \geq 1$. Suppose the following condition is satisfied:
(a) $\lim _{n \rightarrow \infty} \beta_{n}=0$;
(b) $\sum_{n=1}^{\infty} \beta_{n}=\infty$;
(c) $0<s \leq s_{n} \leq k<\left(\frac{q}{C_{q}\|A\|^{q}}\right)^{\frac{1}{q-1}}$.

Then the sequence $\left\{x_{n}\right\}$ and $\left\{u_{n}\right\}$ converges strongly to a point $\Pi_{F(T) \cap \mathcal{F}} \mathcal{u}$.

Proof. If $\alpha_{n}=0$ for all $n \geq 1$ in Theorem 1 , then we obtain the desired conclusion. 
Corollary 2. Let $E_{1}, E_{2}$ be two p-uniformly convex real Banach spaces which are also uniformly smooth and $C$, $Q$ be nonempty closed convex subsets of $E_{1}, E_{2}$, respectively. Let $A: E_{1} \rightarrow E_{2}$ be a bounded linear operator and $A^{*}: E_{2}^{*} \rightarrow E_{1}^{*}$ be the adjoint of $A$. Suppose that the problem (SFP) has a nonempty solution set $\mathcal{F}$. Let $T: C \rightarrow C$ be a right Bregman strongly quasi-nonexpansive mapping such that $F(T)=\widehat{F}(T) \neq \varnothing$ and $F(T) \cap \mathcal{F} \neq \varnothing$. Suppose that $\left\{\alpha_{n}\right\}$ is a sequences in $(0,1)$ such that $\alpha_{n} \leq b<1, a \in\left(0, \frac{1}{2}\right)$. For any fixed $u \in C$, let the sequences $\left\{x_{n}\right\}$ and $\left\{u_{n}\right\}$ be iteratively generated by $u_{0} \in E_{1}$ and

$$
\left\{\begin{array}{l}
x_{n}=\Pi_{C} J_{E_{1}^{*}}^{q}\left(J_{E_{1}}^{p}\left(u_{n}\right)-s_{n} A^{*} J_{E_{2}}^{p}\left(I-P_{Q}\right) A u_{n}\right), \\
u_{n+1}=\Pi_{C} J_{E_{1}^{*}}^{q}\left(\alpha_{n} J_{E_{1}}^{p}(u)+\left(1-\alpha_{n}\right) J_{E_{1}}^{p}\left(T x_{n}\right)\right)
\end{array}\right.
$$

for each $n \geq 1$. Suppose the following conditions are satisfied:

(a) $\lim _{n \rightarrow \infty} \alpha_{n}=0$

(b) $\sum_{n=1}^{\infty} \alpha_{n}=\infty$;

(c) $0<s \leq s_{n} \leq k<\left(\frac{q}{C_{q}\|A\|^{q}}\right)^{\frac{1}{q-1}}$.

Then the sequence $\left\{x_{n}\right\}$ and $\left\{u_{n}\right\}$ converges strongly to a point $\Pi_{F(T) \cap \mathcal{F}} u$.

Proof. If $\beta_{n}=\gamma_{n}=\delta_{n}=0$ for all $n \geq 1$ in Theorem 1, then we obtain the desired conclusion.

Next, we consider the mapping $T: C \rightarrow C$ defined by $T=T_{N} \circ T_{N-1} \circ \cdots \circ T_{1}$, where $T_{i}$ for each $i=1,2, \cdots, N$ is a right Bregman strongly quasi-nonexpansive mapping on $E$. Using the results in [10], we have the following:

Corollary 3. Let $E_{1}, E_{2}$ be two p-uniformly convex real Banach spaces which are also uniformly smooth and $C$, $Q$ be nonempty closed convex subsets of $E_{1}, E_{2}$, respectively. Let $A: E_{1} \rightarrow E_{2}$ be a bounded linear operator and $A^{*}: E_{2}^{*} \rightarrow E_{1}^{*}$ be the adjoint of $A$. Suppose that the problem (SFP) has a nonempty solution set $\mathcal{F}$. Let $T=T_{N} \circ T_{N-1} \circ \cdots \circ T_{1}$, where $T_{i}: C \rightarrow C$ for each $i=1,2, \cdots N$ be a finite family of right Bregman strongly quasi-nonexpansive mappings such that $F\left(T_{i}\right)=\widehat{F}\left(T_{i}\right) \neq \varnothing$ and $\left(\bigcap_{i=1}^{N} F\left(T_{i}\right)\right) \cap \mathcal{F} \neq \varnothing$. Suppose that $\left\{\alpha_{n}\right\},\left\{\beta_{n}\right\},\left\{\gamma_{n}\right\}$ and $\left\{\delta_{n}\right\}$ are the sequences in $[0,1)$ such that $\alpha_{n}+\beta_{n}+\gamma_{n}+\delta_{n}=1, \alpha_{n} \leq b<1$, $\left(1-\alpha_{n}\right) a<\gamma_{n}<\delta_{n}, a \in\left(0, \frac{1}{2}\right)$. For any fixed $u \in C$, let the sequences $\left\{x_{n}\right\}$ and $\left\{u_{n}\right\}$ be iteratively generated by $u_{0} \in E_{1}$ and

$$
\left\{\begin{array}{l}
x_{n}=\Pi_{C} J_{E_{1}^{*}}^{q}\left(J_{E_{1}}^{p}\left(u_{n}\right)-s_{n} A^{*} J_{E_{2}}^{p}\left(I-P_{Q}\right) A u_{n}\right), \\
u_{n+1}=\Pi_{C} J_{E_{1}^{*}}^{q}\left(\alpha_{n} J_{E_{1}}^{p}(u)+\left(1-\alpha_{n}\right)\left(\beta_{n} J_{E_{1}}^{p}(u)+\gamma_{n} J_{E_{1}}^{p}\left(x_{n}\right)+\delta_{n} J_{E_{1}}^{p}\left(T x_{n}\right)\right)\right)
\end{array}\right.
$$

for each $n \geq 1$. Suppose the following conditions are satisfied:

(a) $\lim _{n \rightarrow \infty} \alpha_{n}=0$;

(b) $\sum_{n=1}^{\infty} \alpha_{n}=\infty$;

(c) $0<s \leq s_{n} \leq k<\left(\frac{q}{C_{q}\|A\|^{q}}\right)^{\frac{1}{q-1}}$.

Then the sequence $\left\{x_{n}\right\}$ and $\left\{u_{n}\right\}$ converges strongly to a point $\Pi_{F(T) \cap \mathcal{F}} u$.

Proof. If $T=T_{N} \circ T_{N-1} \circ \cdots \circ T_{1}$ in Theorem 1, then we obtain the desired conclusion.

Corollary 4. Let $H_{1}, H_{2}$ be two real Hilbert spaces and $C, Q$ be nonempty closed convex subsets of $H_{1}$, $H_{2}$, respectively. Let $A: H_{1} \rightarrow H_{2}$ be a bounded linear operator and $A^{*}: H_{2} \rightarrow H_{1}$ be the adjoint of $A$. 
Suppose that the problem (SFP) has a nonempty solution set $\mathcal{F}$. Let $T: C \rightarrow C$ be a right Bregman strongly quasi-nonexpansive mapping such that $F(T)=\widehat{F}(T) \neq \varnothing, I-T$ is demiclosed at zero and $F(T) \cap \mathcal{F} \neq \varnothing$. Suppose that $\left\{\alpha_{n}\right\},\left\{\beta_{n}\right\},\left\{\gamma_{n}\right\}$ and $\left\{\delta_{n}\right\}$ are the sequences in $[0,1)$ such that $\alpha_{n}+\beta_{n}+\gamma_{n}+\delta_{n}=1$, $\alpha_{n} \leq b<1,\left(1-\alpha_{n}\right) a<\gamma_{n}<\delta_{n}, a \in\left(0, \frac{1}{2}\right)$. For any fixed $u \in C$, let the sequences $\left\{x_{n}\right\}$ and $\left\{u_{n}\right\}$ be iteratively generated by $u_{0} \in E_{1}$ and

$$
\left\{\begin{array}{l}
x_{n}=P_{C}\left(u_{n}-s_{n} A^{*}\left(I-P_{Q}\right) A u_{n}\right) \\
u_{n+1}=P_{C}\left(\alpha_{n} u+\left(1-\alpha_{n}\right)\left(\beta_{n} u+\gamma_{n} x_{n}+\delta_{n} T x_{n}\right)\right)
\end{array}\right.
$$

for each $n \geq 1$. Suppose the following conditions are satisfied:

(a) $\lim _{n \rightarrow \infty} \alpha_{n}=0 ;$
(b) $\sum_{n=1}^{\infty} \alpha_{n}=\infty ;$
(c) $0<s \leq s_{n} \leq k<\frac{2}{\|A\|^{2}}$.

Then the sequence $\left\{x_{n}\right\}$ and $\left\{u_{n}\right\}$ converges strongly to a point $\Pi_{F(T) \cap \mathcal{F}} u$.

Proof. Let $E=H$ in Theorem 1. Since the duality mappings $J_{E_{1}^{*},}^{q} J_{E_{1}}^{p}$ and $J_{E_{2}}^{p}$ are the identity mapping in a Hilbert space $H$, from Theorem 1 , we obtain the desired conclusion.

Corollary 5. Let $H_{1}, H_{2}$ be two real Hilbert spaces and $C, Q$ be nonempty closed convex subsets of $H_{1}$, $H_{2}$, respectively. Let $A: H_{1} \rightarrow H_{2}$ be a bounded linear operator and $A^{*}: H_{2} \rightarrow H_{1}$ be the adjoint of $A$. Suppose that the problem (SFP) has a nonempty solution set $\mathcal{F}$. Let $T: C \rightarrow C$ be a right Bregman strongly quasi-nonexpansive mapping such that $F(T)=\widehat{F}(T) \neq \varnothing, I-T$ is demiclosed at zero and $F(T) \cap \mathcal{F} \neq \varnothing$. Suppose that $\left\{\beta_{n}\right\},\left\{\gamma_{n}\right\}$ and $\left\{\delta_{n}\right\}$ are sequences in $[0,1)$ such that $\beta_{n}+\gamma_{n}+\delta_{n}=1, \beta_{n} \leq b<1$, $\left(1-\beta_{n}\right) a<\gamma_{n}<\delta_{n}, a \in\left(0, \frac{1}{2}\right)$. For any fixed $u \in C$, let the sequences $\left\{x_{n}\right\}$ and $\left\{u_{n}\right\}$ be iteratively generated by $u_{0} \in E_{1}$ and

$$
\left\{\begin{array}{l}
x_{n}=P_{C}\left(u_{n}-s_{n} A^{*}\left(I-P_{Q}\right) A u_{n}\right), \\
u_{n+1}=P_{C}\left(\beta_{n} u+\gamma_{n} x_{n}+\delta_{n} T x_{n}\right)
\end{array}\right.
$$

for each $n \geq 1$. Suppose the following conditions are satisfied:
(a) $\lim _{n \rightarrow \infty} \beta_{n}=0$;
(b) $\sum_{n=1}^{\infty} \beta_{n}=\infty$;
(c) $0<s \leq s_{n} \leq k<\frac{2}{\|A\|^{2}}$.

Then the sequence $\left\{x_{n}\right\}$ and $\left\{u_{n}\right\}$ converges strongly to a point $\Pi_{F(T) \cap \mathcal{F}} u$.

Proof. Let $E=H$ and $\alpha_{n}=0$ for each $n \geq 1$. Since the duality mappings $J_{E_{1}^{*}}^{q} J_{E_{1}}^{p}$ and $J_{E_{2}}^{p}$ are the identity mapping in a Hilbert space $H$, from Theorem 1 , we obtain the desired conclusion.

Remark 1. A prototype for the sequences $\left\{\alpha_{n}\right\},\left\{\beta_{n}\right\},\left\{\gamma_{n}\right\},\left\{\delta_{n}\right\}$ and $\left\{s_{n}\right\}$ in Theorem 1 are as follows:

$$
\alpha_{n}=\frac{1}{n+1}, \quad \beta_{n}=1-\frac{2 a n+1}{n+1}, \quad \gamma_{n}=\frac{\frac{1}{2} a n}{n+1}, \quad \delta_{n}=\frac{\frac{3}{2} a n}{n+1}, \quad a \in\left(0, \frac{1}{2}\right)
$$

and

$$
s_{n}=\left(\frac{n}{n+1}\right)\left(\frac{q}{C_{q}\|A\|^{q}}\right)^{\frac{1}{q-1}}
$$

for each $n \geq 0$. 


\section{Some Numerical Examples}

In this section, we present some preliminary numerical results to illustrate the main result, Theorem 1. All codes were written in Matlab 2013b and run on Sumsung i-3 Core laptop.

Example 1. We find a numerical example in $\left(\mathbb{R}^{3},\|\cdot\|_{2}\right)$ of the problem considered in Theorem 1 of the previous section. Now, take

$$
C:=\left\{x=\left(x_{1}, x_{2}, x_{3}\right) \in \mathbb{R}^{3}:\langle a, x\rangle \geq b\right\},
$$

where $a=(2,-6,1)$ and $b=-4$. Then we have

$$
P_{C}(x)=\frac{b-\langle a, x\rangle}{\|a\|_{2}^{2}} a+x .
$$

Let $Q:=\left\{x=\left(x_{1}, x_{2}, x_{3}\right) \in \mathbb{R}^{3}:\langle c, x\rangle=d\right\}$, where $c=(3,5,7)$ and $d=2$. Then we have

$$
\Pi_{Q}(x)=P_{Q}(x)=\max \left\{0, \frac{d-\langle c, x\rangle}{\|c\|_{2}^{2}}\right\} c+x .
$$

Suppose that the mapping $T$ in Theorem 1 is defined as $T:=P_{C}$, the metric projection on $C$. Then the problem considered in Theorem 1 reduces to the problem:

$$
\text { Find } x \in F(T) \cap C(=C) \text { such that } A x \in Q \text {. }
$$

Let $\mathcal{F}$ denote the set of solutions of the problem (48) with $\mathcal{F} \neq \varnothing$. Furthermore, let

$$
\alpha_{n}=\frac{1}{n+1}, \beta_{n}=\frac{n}{8(n+1)}, \gamma_{n}=\frac{n}{8(n+1)}, \delta_{n}=\frac{3 n}{8(n+1)}, A=\left(\begin{array}{ccc}
4 & -6 & -8 \\
-5 & 1 & -5 \\
-8 & -5 & 4
\end{array}\right) \text {. }
$$

Then our iterative processes (20) becomes

$$
\left\{\begin{array}{l}
x_{n}=P_{C}\left(u_{n}-s_{n} A^{T}\left(I-P_{Q}\right) A u_{n}\right), \\
u_{n+1}=P_{C}\left[\frac{u}{n+1}+\left(1-\frac{1}{n+1}\right)\left(\frac{n}{2(n+1)} u+\frac{n}{8(n+1)} x_{n}+\frac{3 n}{8(n+1)} P_{C} x_{n}\right)\right]
\end{array}\right.
$$

for each $n \geq 1$. Now, we make different choices of $u, u_{1}, s_{n}$ and take $\frac{\left\|x_{n+1}-x_{n}\right\|}{\left\|x_{2}-x_{1}\right\|}<10^{-2}$ as our stopping criterion.

Case I. Take $u=(1,1,1), u_{1}=(3,0,4)$ and $s_{n}=0.0137$. Then we have the numerical analysis tabulated in Table 1 and show in Figure 1.

Table 1. Example 1, case I.

\begin{tabular}{ccccc}
\hline$s_{\boldsymbol{n}}$ & Time Taken & Number of Iterations & $\left\|x_{\boldsymbol{n}+\mathbf{1}}-x_{\boldsymbol{n}}\right\|_{\mathbf{2}}$ & $\left\|\boldsymbol{u}_{\boldsymbol{n}+\mathbf{1}}-\boldsymbol{u}_{\boldsymbol{n}}\right\|_{\mathbf{2}}$ \\
\hline & 2 & 0.6758 & 4.2169 \\
& 3 & 0.0122 & 0.0453 \\
4 & 5 & 0.0177 & 0.0497 \\
0.0137 & \multirow{3}{*}{0.0400} & 6 & 0.0158 & 0.0265 \\
& & 7 & 0.0146 & 0.0194 \\
& & 8 & 0.0130 & 0.0154 \\
& 9 & 0.0113 & 0.0127 \\
& 10 & 0.0098 & 0.0107 \\
& & 11 & 0.0085 & 0.0091 \\
& & 0.0074 & 0.0079 \\
\hline
\end{tabular}




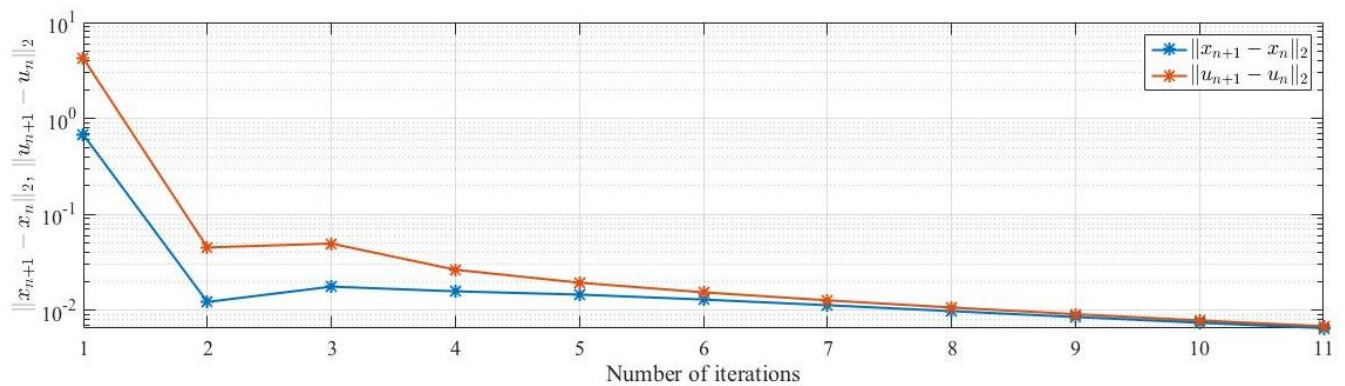

Figure 1. Example 1, case I.

Case II. Take $u=(1,1,1), u_{1}=(3,0,4)$ and $s_{n}=0.0001$. Then we have the numerical analysis tabulated in Table 2 and show in Figure 2.

Table 2. Example 1, case II.

\begin{tabular}{ccccc}
\hline$s_{n}$ & Time Taken & Number of Iterations & $\left\|x_{n+1}-x_{n}\right\|_{2}$ & $\left\|u_{n+1}-u_{n}\right\|_{2}$ \\
\hline & & 2 & 1.7588 & 3.9690 \\
& & 4 & 0.1950 & 0.1968 \\
& & 5 & 0.0181 & 0.0182 \\
0.0001 & \multirow{4}{*}{0.0400} & 6 & 0.0265 & 0.0266 \\
& & 7 & 0.0318 & 0.0320 \\
& & 8 & 0.0303 & 0.0305 \\
& & 10 & 0.0271 & 0.0273 \\
& & 11 & 0.0237 & 0.0239 \\
& & & 0.0207 & 0.0209 \\
& & & 0.0181 & 0.0182 \\
\hline
\end{tabular}

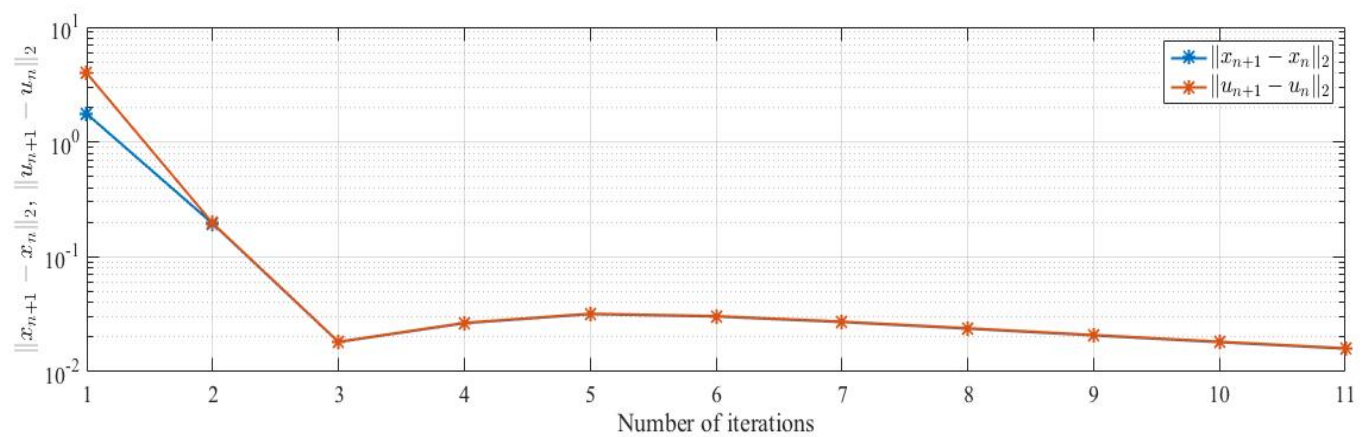

Figure 2. Example 1, case II.

Case III. Take $u=(1,1,1), u_{1}=(3,0,4)$ and $s_{n}=0.0000001$. Then we have the numerical analysis tabulated in Table 3 and show in Figure 3.

Table 3. Example 1, case III.

\begin{tabular}{ccccc}
\hline$s_{n}$ & Time Taken & Number of Iterations & $\left\|x_{n+1}-x_{n}\right\|_{2}$ & $\left\|u_{n+1}-u_{n}\right\|_{2}$ \\
\hline & 2 & 1.7720 & 3.9674 \\
& & 4 & 0.1969 & 0.1969 \\
0.0000001 & \multirow{3}{*}{0.0400} & 5 & 0.0179 & 0.0179 \\
& & 6 & 0.0272 & 0.0272 \\
& & 7 & 0.0326 & 0.0326 \\
& & 0.0311 & 0.0311 \\
& & 10 & 0.0278 & 0.0278 \\
& & 0.0244 & 0.0244 \\
& & 0.0213 & 0.0213 \\
& & 0.0186 & 0.0186 \\
\hline
\end{tabular}




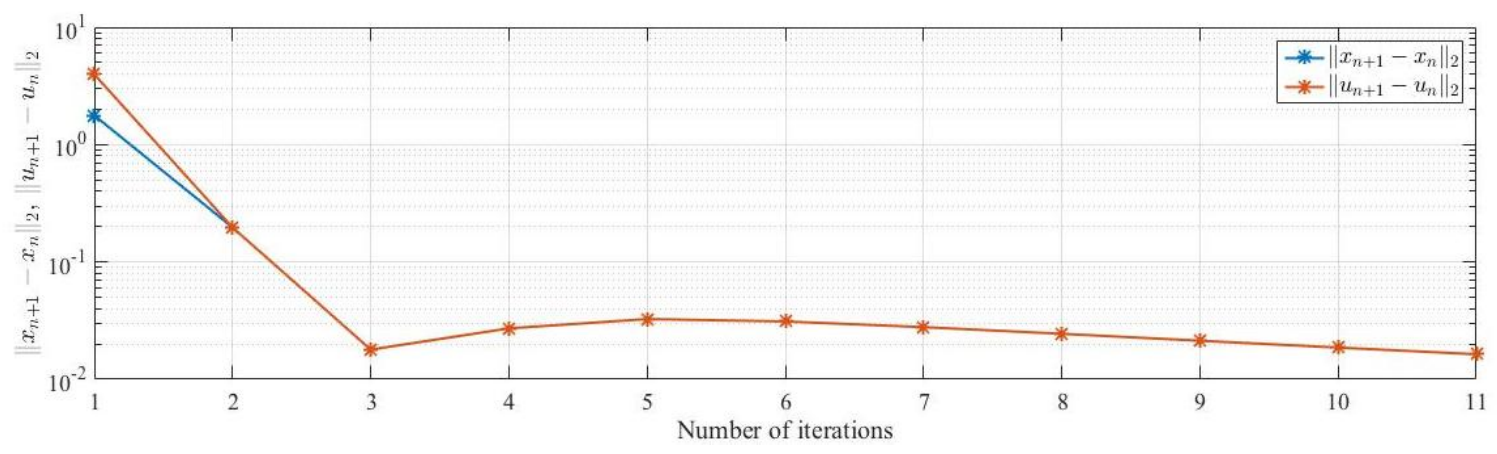

Figure 3. Example 1, case III.

Remark 2. We make the following comments from Example 1. We observe that different choices of $s_{n}$ has no effect in terms of number of iterations obtained and the time taken for the convergence of our algorithm.

\section{Conclusions}

Our iterative processes can be used for finding a common element of the solution set $\mathcal{F}$ of the split feasibility problem and the fixed point set $F(T)$ of a right Bregman strongly quasi-nonexpansive mapping $T$ in $p$-uniformly convex Banach spaces, which are also uniformly smooth.

Acknowledgments: The work was partially supported by the Higher Education Research Promotion and National Research University Project of Thailand, Office of the Higher Education Commission (NRU59 Grant No. 59000399). The first author thanks for the support of Petchra Pra Jom Klao Doctoral Scholarship (Grant No. 21/2558) for Ph.D. student of King Mongkut's University of Technology Thonburi (KMUTT). Moreover, this research work was financially supported by King Mongkuts University of Technology North Bangkok. Contract No. KMUTNB-60-ART-084.

Author Contributions: P.K. and Y.J.C. conceived and designed the direction of research; P.K. and A.P. wrote the paper and also derived the main theorems and consequences together with proofs; P.T. and A.P. analyzed the data and advised and revised the final contents in the paper; all authors contributed equally and significantly in writing this article.

Conflicts of Interest: The authors declare no conflict of interest.

\section{References}

1. Censor, Y.; Elfving, T. A multiprojection algorithm using Bregman projections in a product space. Numer. Algorithms 1994, 8, 221-239.

2. Byrne, C. Iterative oblique projection onto convex sets and the split feasibility problem. Inverse Probl. 2002, $18,441-453$.

3. Kumam, W.; Witthayarat, U.; Wattanawitoon, K.; Suantai, S.; Kumam, P. Convergence theorem for equilibrium problem and bregman strongly nonexpansive mappings in Banach spaces. Optim. J. Math. Progr. Oper. Res. 2016, 65, 265-280.

4. Witthayarat, U.; Wattanawitoon, K.; Kumam, P. Iterative scheme for system of equilibrium problems and Bregman asymptotically quasi-nonexpansive mappings in Banach spaces. J. Inf. Optim. Sci. 2016, 37, 321-342.

5. Kumam, W.; Sunthrayuth, P.; Phunchongharn, P.; Akkarajitsakul, K.; Sa Ngiamsunthorn, P.; Kumam, P. A new multi-step iterative algorithm for approximating common fixed points of a finite family of multi-valued bregman relatively nonexpansive mappings. Algorithms 2016, 9, 37.

6. Shehu, Y.; Mewomo, O.T.; Ogbuisi, F.U. Further investigation into approximation of a common solution of fixed point problems and split feasibility problems. Acta Math. Sci. 2016, 36, 913-930.

7. Schöpfer, F. Iterative Regularization Method for the Solution of the Split Feasibility Problem in Banach Spaces. Dissertation zur Erlangung des Grades des Doktors der Naturwissenschaften, NaturwissenschaftlichTechnischen Fakultäten, Universität des Saarlandes, Saarbrücken, Germany, 2007.

8. Schopfer, F.; Schuster, T.; Louis, A.K. An iterative regularization method for the solution of the split feasibility problem in Banach spaces. Inverse Probl. 2008, 24, 5. 
9. Wang, F. A new algorithm for solving the multiple-sets split feasibility problem in Banach spaces. Numer. Funct. Anal. Optim. 2014, 35, 99-110.

10. Zegeye, H.; Shahzad, N. Convergence theorems for right bregman strongly nonexpansive mappings in reflexive banach spaces. Abstr. Appl. Anal. 2014, 2014, 584395.

11. Takahashi, W. Nonlinear Functional Analysis-Fixed Point Theory and Applications; Yokohama Publishers Inc.: Yokohama, Japan, 2000. (In Japanese)

12. Dunford, N.; Schwartz, J.T.; Linear Operators I; Wiley Interscience: New York, NY, USA, 1958.

13. Bregman, L.M. The relaxation method for finding common fixed points of convex sets and its application to the solution of problems in convex programming. USSR Comput. Math. Math. Phys. 1976, 7, 200-217.

14. Goebel, K.; Reich, S. Uniform Convexity, Hyperbolic Geometry, and Nonexpansive Mappings; Marcel Dekker: New York, NY, USA, 1984.

15. Censor, Y.; Lent, A. An iterative row-action method for interval convex programming. J. Optim. Theory Appl. 1981, 34, 321-353.

16. Alber, Y.I. Metric and generalized projection operator in Banach spaces: Properties and applications. In Theory and Applications of Nonlinear Operators of Accretive and Monotone Type; Lecture Notes in Pure and Applied Mathematics; Dekker: New York, NY, USA, 1996; Volume 178, pp. 15-50.

17. Phelps, R.P. Convex Functions, Monotone Operators, and Differentiability, 2nd ed.; Springer: Berlin, Germany, 1993; Volume 1364.

18. Kohsaka, F.; Takahashi, W. Proximal point algorithms with Bregman functions in Banach spaces. J. Nonlinear Convex Anal. 2005, 6, 505-523.

19. Shehu, Y.; Ogbuisi, F.U.; Iyiola, O.S. Convergence analysis of an iterative algorithm for fixed point problems and split feasibility problems in certain Banach spaces. J. Math. Program. Oper. Res. 2016, 65, doi:10.1080/02331934.2015.1039533.

20. Shehu, Y.; Iyiola, O.S.; Enyi, C.D. An iterative algorithm for split feasibility problems and fixed point problems in Banach spaces. Numer. Algorithms 2016, 72, 835-864.

21. Reich, S. A weak convergence theorem for the alternating method with Bregman distances. In Theory and Applications of Nonlinear Operators; Marcel Dekker: New York, NY, USA, 1996; pp. 313-318.

22. Reich, S.; Sabach, S. Two strong convergence theorems for a proximal method in reflexive Banach spaces. Numer. Funct. Anal. Optim. 2010, 31, 22-44.

23. Martin-Marquez, V.; Reich, S.; Sabach, S. Right Bregman nonexpansive operators in Banach spaces. Nonlinear Anal. 2012, 75, 5448-5465.

24. Martin-Marquez, V.; Reich, S.; Sabach, S. Existence and approximation of fixed points of right Bregman nonexpansive operators. In Computational and Analytical Mathematics; Springer Proceedings in Mathematics \& Statistics; Springer: New York, NY, USA, 2013; pp. 501-520.

25. Mainge, P.E. Strong convergence of projected subgradient methods for nonsmooth and nonstrictly convex minimization. Set-Valued Anal. 2008, 16, 899-912.

26. Xu, H.K. Iterative algorithms for nonlinear operators. J. Lond. Math. Soc. 2002, 66, 240-256.

(C) 2016 by the authors; licensee MDPI, Basel, Switzerland. This article is an open access article distributed under the terms and conditions of the Creative Commons Attribution (CC-BY) license (http://creativecommons.org/licenses/by/4.0/). 